Vol. 18 (2009): 152-159.

\title{
Use of secondary somatic embryos promotes genetic fidelity in cryopreservation of cocoa (Theobroma cacao L.)
}

Jong-Yi Fang ${ }^{1}$, Andy Wetten ${ }^{2 *}$, Raphael Adu-Gyamfi², Mike Wilkinson ${ }^{3}$ and Carlos Rodriguez-Lopez ${ }^{3}$

${ }^{1}$ National Pingtung University of Science and Technology, Department of Tropical Agriculture and International Cooperation, Neipu, Pingtung, Taiwan 91201.

${ }^{2}$ University of Reading, School of Biological Sciences, Plant Science Laboratories, Whiteknights, Reading, PO Box 221, RG6 6AS, UK.

${ }^{3}$ University of Wales Aberystwyth, Institute of Biological Sciences, Edward Llwyd Building,

Penglais, Ceredigion SY23 3DA, UK.

*email: a.c.wetten@reading.ac.uk

The inability to conserve cocoa (Theobroma cacao L.) germplasm via seed storage and the vulnerability of field collections make the establishment of cryopreserved genebanks for the crop a priority. An effective encapsulation-dehydration based cryopreservation system has been developed for cocoa but because the somatic embryos used for freezing arise after a protracted period of callus culture there is concern about maintenance of genetic fidelity during the process. Microsatellite markers for seven of the 10 cocoa linkage groups were used to screen a population of 189 primary somatic embryo-derived emblings and the 43 secondary somatic embryos they gave rise to. Of the primary somatic embryos, $38.1 \%$ exhibited polymorphic microsatellite profiles while for secondary somatic embryos the frequency was $23.3 \%$. The same microsatellite markers used to screen another population of 44 secondary somatic embryos cryopreserved through encapsulation-dehydration revealed no polymorphisms. Scanning electron microscopy showed the secondary somatic embryos were derived from cotyledonary epidermal cells rather than callus. The influence of embryo ontogeny on somaclonal variation is discussed.

Key-words: Cocoa, Theobroma cacao, somatic embryogenesis, somaclonal variation, cryopreservation 
Vol. 18 (2009): 152-159.

\section{Introduction}

A drought in 2007 in the Ivory Coast, the world's largest cocoa producer, sent futures in the crop to their highest levels since 2003. Other factors contributing to the price climb include the spread of cocoa swollen shoot virus in West Africa and consumers' growing taste for dark chocolate (which has a higher concentration of cocoa solids). Such pressures for improved yield and stress and disease resistance highlight the importance of preserving securely the diversity of the species for future breeding goals. Though valuable, field collections are prone to losses through climatic catastrophes and the action of pests and diseases. These risks, together with the recalcitrant nature of cocoa seed with regard to storage, makes the establishment of a cryopreserved collection of key cocoa germplasm a logical safeguard. To this end over 600 accessions of cocoa are being cryopreserved at Reading University through the encapsulation-dehydration of floral-derived somatic embryos (SEs) (Fang et al. 2004). This procedure involves the rapid cooling of the prolific secondary SEs obtained from cultured cotyledonary explants of primary SEs.

With a view to maintaining the genetic fidelity of cryopreserved plant germplasm, shoot tip culture has become the propagation system of choice (eg. Turner et al. 2001, Wilkinson et al. 2003). In the case of cocoa, in vitro shoot regeneration has proved problematic (Figueria and Janick 1994), though rapid clonal multiplication can be achieved via SEs derived from the floral tissues. Primary SEs can be induced from cocoa petal bases and staminodes (Li et al. 1998) and multiplication rates can be further increased through the production of secondary SEs from cotyledonary explants of primary SEs (Maximova et al. 2002). However, protracted callus phases such as those necessary for somatic embryogenesis are linked to induction of somaclonal variation and cocoa primary SEs screened with microsatellite markers were found to exhibit mutations (Rodriguez-Lopez et al. 2004). Cryopreservation of plant germplasm in the form of meristematic explants has been reported to minimise genetic mutation (Wilkinson et al. 2003, Liu et al. 2008) but studies of SE based cryopreservation have proved less conclusive (e.g. Dixit et al. 2003).
Microsatellite analysis has been used in several studies evaluating the clonal fidelity of plants recovered from cryopreservation (e.g. Harding and Benson 2001, Richards et al. 2004). Microsatellites are tandem repeats of short DNA sequence motifs (1-6 bases long). They appear to be ubiquitous among organisms and, though initially thought to be randomly distributed throughout genomes, Fujimori et al. (2003) identified the plant specific feature of a gradient in microsatellite density along the direction of transcription, suggesting that microsatellites located at high frequency in the 5'-flanking regions of plant genes could act as factors in regulating gene expression.

Cocoa $(2 n=20)$ has a small genome containing $3.88 \times 10^{8}$ bp (Lanaud et al. 1992), corresponding to approximately twice that of Arabidopsis thaliana L., and the high density of cocoa microsatellites now mapped facilitates detection of modifications to this genome (Lanaud et al. 1999). These microsatellites provide useful tools for the discrimination of DNA polymorphisms between somaclones since they demonstrate high locus specificity, high polymorphism and co-dominance (Risterucci et al. 2000). Microsatellites were therefore adopted here for somaclonal variation screening.

In this study microsatellite markers have been used to establish the frequency of somaclonal variation in a population of primary SEs and their descendent secondary SEs. The same markers have also been utilised to assess somaclonal variation in secondary SEs exposed to progressive stages of an encapsulation-dehydration cryopreservation protocol. Scanning Electron Microscopy (SEM) has been used to characterise the tissue origin of the secondary SEs used for cryopreservation.

\section{Material and Methods}

\section{Plant material}

A total of 189 primary SEs were initiated on floral staminodes harvested from individual cocoa trees (genotypes LCT EEN 162/S-10101, LCT EEN 
Fang, J-Y. et al. Genetic fidelity of cryopreserved cocoa somatic embryos

37/I, SC 3 and SIAL 93) at Reading University's International Intermediate Quarantine collection as per Li et al. (1998). Cotyledonary explants from these primary SEs were used for the initiation of secondary SEs (Maximova et al. 2002). The bulk of embryo production for this population took place between 12 and 26 weeks after culture initiation.

An additional population of secondary SEs was initiated from the genotype AMAZ 12 with the majority of embryos appearing between 14 to 18 weeks in culture. Fourty four of these secondary SEs were used for cryopreservation through encapsulation-dehydration (Fang et al. 2004). Subsets of 11 secondary SEs were sampled during cryopreservation at intervals following (a) secondary SE initiation, (b) sucrose pretreatment, (c) dehydration over silica and (d) thawing after storage in liquid nitrogen for $24 \mathrm{~h}$. Embryos were sampled for this study irrespective of morphology.

\section{DNA extraction and amplification}

Two tissue samples (either true leaf or cotyledon) were taken from each somatic embryo-derived plantlet (embling). To screen for intra-ortet variation 20 individual samples were taken from dispersed leaves of each explant source tree of the genotypes LCT EEN 162/S-10101, LCT EEN 37/I, SC 3 and SIAL 93. Samples of approximately 50 $\mathrm{mg}$ of frozen tissue were loaded in microtubes each with a tungsten bead and macerated by agitation in a Retsch MM300 Mixer Mill. Total genomic DNA extraction was performed using the DNeasy $^{\mathrm{TM}} 96$ plant kit (Qiagen Ltd., UK) which allows for the simultaneous isolation of DNA from 192 samples.

Eighteen microsatellite primers were tested on the five parental clones following the PCR protocol of Lanaud et al. (1999). PCR products $(5 \mu 1)$ were test fractionated on agarose gels and those yielding consistently strong amplifications were selected for further use. MicrosatellitePCR analysis was performed with one primer 5' end-labelled using 6-FAM (blue), HEX (green) or NED (yellow). Labelled PCR products were diluted $1 / 10$ in nanopure water and $1 \mu \mathrm{l}$ of ROX/ HiDi mix. Samples were heat-denatured at $95^{\circ} \mathrm{C}$ for 3-5min and snap-cooled on ice for $2 \mathrm{~min}$. Samples were fractionated on an ABI PRISM 3100 at $3 \mathrm{kV}$ for $22 \mathrm{~s}$ and at $15 \mathrm{kV}$ for $45 \mathrm{~min}$ at $60^{\circ} \mathrm{C}$. Resultant profiles were analyzed using Genotyper 3.7NT software (Applera Corporation).

\section{Scanning Electron Microscopy}

SEM was used to assess the tissue origin of secondary SEs initiated from cotyledonary explants. Tissues were fixed for 2 hours in $2 \%$ paraformaldehyde / $2.5 \%$ glutaraldehyde prepared in $25 \mathrm{mM}$ piperazine-1,4-bis(2-ethanesulfonic acid) (PIPES) buffer ( $\mathrm{pH}$ 6.7). After two washes in PIPES buffer, samples were dehydrated through an acetone series and dried in a Bal-Tec CPD 030 critical point dryer. Samples were mounted and sputter coated (Edwards S150B) with gold for $2 \mathrm{~min}$ at $25 \mathrm{~mA}$ and imaged with the FEI Quanta 600F SEM at $20 \mathrm{kV}$ using the high vacuum mode.

\section{Results}

\section{Microsatellite selection}

Ten of the original 18 microsatellites were selected as giving consistent profiles for the five genotypes under test (Table 1). Primers mTcCIR1, 2, 3, 6, $7,8,10,18,25$ and 26 which mapped to seven of cocoa's 10 linkage groups, were used for PCR screening for variation within the primary SE donor trees and revealed no polymorphic profiles within any genotype. Three independent DNA extractions from leaves of the donor tree AMAZ 12 also failed to reveal any polymorphisms when screened with the same 10 microsatellite primers. 
Vol. 18 (2009): 152-159.

Table 1. Microsatellite allele sizes in base pairs (bp) for five cocoa parental genotypes and derived somatic embryos screened for somaclonal variation. Expected allele sizes were obtained from Lanaud et al. (1999) and Risterucci et al. (2000).

\begin{tabular}{|c|c|c|c|c|c|c|c|}
\hline \multirow{2}{*}{$\begin{array}{l}\text { Linkage group } \\
\text { (Locus) }\end{array}$} & \multirow[b]{2}{*}{ Repeat structure } & \multicolumn{5}{|c|}{ GENOTYPE } & \multirow{2}{*}{$\begin{array}{l}\text { Expected } \\
\text { size (bp) }\end{array}$} \\
\hline & & $\begin{array}{c}\text { AMAZ } \\
12 \\
\end{array}$ & $\begin{array}{c}\text { LCTEEN } \\
\text { 37I } \\
\end{array}$ & $\begin{array}{l}\text { LCTEEN } \\
162 / 1010 \\
\end{array}$ & $\mathrm{SC} 3$ & SIAL93 & \\
\hline 2 (mTcCIR3) & $(\mathrm{CT})_{20}(\mathrm{TA})_{21}$ & 224,226 & 226 & 226 & 232,240 & 217,224 & 249 \\
\hline $4(\mathrm{mTcCIR} 18)$ & $(\mathrm{GA})_{12}$ & 331,353 & 342 & 337,353 & 334,344 & 335,344 & 345 \\
\hline 5 (mTcCIR10) & $(\mathrm{TG})_{13}$ & 200,202 & 203 & 205,207 & 201,203 & 203,205 & 208 \\
\hline 5 (mTcCIR2) & $(\mathrm{GA})_{3} \mathrm{~N}_{5}(\mathrm{AG})_{2} \mathrm{GG}(\mathrm{AG})_{4}$ & 238,252 & 252 & 238,252 & 242,256 & 238,252 & 254 \\
\hline 6 (mTcCIR6) & $(\mathrm{TG})_{7}(\mathrm{GA})_{13}$ & 225,237 & 231,236 & 225 & 223,230 & 224,230 & 231 \\
\hline $6(\mathrm{mTcCIR} 25)$ & $(\mathrm{CT})_{21}$ & 131,147 & 133,135 & 128,129 & 132,152 & 146,165 & 153 \\
\hline 7 (mTcCIR7) & $(\mathrm{GA})_{11}$ & 159,161 & 154,160 & 158 & 158,162 & 158 & 160 \\
\hline 8 (mTcCIR1) & $(\mathrm{CT})_{14}$ & 126,132 & 132,138 & 126 & 132,138 & 138 & 143 \\
\hline 8 (mTcCIR26) & $(\mathrm{TC})_{9} \mathrm{C}(\mathrm{CT})_{4} \mathrm{TT}(\mathrm{CT})_{11}$ & 300,303 & 289,294 & 294,302 & 294,307 & 296,300 & 298 \\
\hline 9 (mTcCIR8) & $(\mathrm{TC})_{5} \mathrm{TT}(\mathrm{TC})_{17} \mathrm{TTT}(\mathrm{CT})_{4}$ & 287,293 & 290,292 & 286 & 287,302 & 286,290 & 301 \\
\hline
\end{tabular}

\section{Primary vs. secondary somatic embryogenesis}

The population of non-cryopreserved SEs exhibited a degree of microsatellite variation within each of the four genotypes tested (Table 2). Observed polymorphisms could be divided into three categories: 1) allele loss; 2) slippage of alleles; and 3) generation of novel alleles (Fig. 1). Of these polymorphic categories, the largest was 'allele loss' (58.8\%). Primary SEs in this cohort exhibited off-type profiles at a frequency of $38.1 \%$. The same microsatellite primers showed only $23.3 \%$ of the descendent secondary SEs to have aberrant profiles. Of the 32 true-to-type secondary SEs, 27 were derived from true-to-type primary SEs, three arose from off-type SEs and two were from SEs unavailable for analysis. As for the 11 off-type secondary SEs, one was

Table 2. Somaclonal variation detected during cocoa primary and secondary somatic embryogenesis using 10 microsatellite markers. The total number of screened samples is shown in brackets for each genotype. Genotype 'SIAL 93' did not produce secondary embryos (-). None of the 44 cryopreserved 'AMAZ 12' secondary somatic embryos screened with the same markers exhibited somaclonal variation.

\begin{tabular}{lccc}
\hline Genotype & Primary off-types & Secondary off-types & Total \\
\hline LCT EEN 162/S-10101 & $36.2 \%(149)$ & $30 \%(30)$ & $35.2 \%$ \\
LCT EEN 37/I & $40 \%(10)$ & $33 \%(3)$ & $38.5 \%$ \\
SC 3 & $40 \%(10)$ & $0 \%(10)$ & $20 \%$ \\
SIAL 93 & $50 \%(20)$ & - & $50 \%$ \\
Total & $38.1 \%$ & $23.3 \%$ & $35.3 \%$ \\
\hline
\end{tabular}




\section{AGRICULTURAL AND FOOD SCIENCE}

Fang, J-Y. et al. Genetic fidelity of cryopreserved cocoa somatic embryos

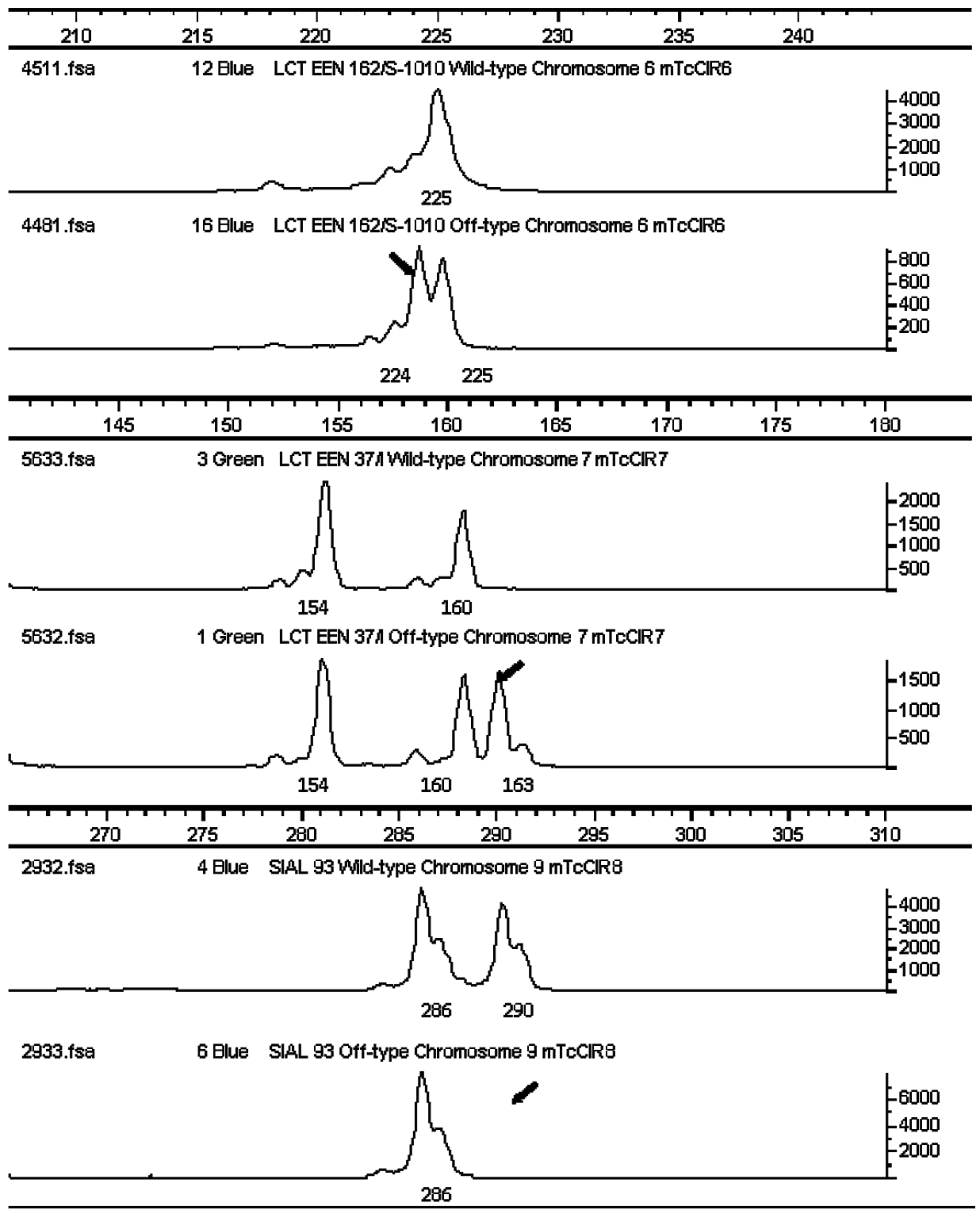

Fig. 1. Parental and example polymorphic profiles for microsatellite markers mTcCIR6, 7 and 8 (from chromosomes 6 , 7 and 9) in primary somatic embryos derived from cocoa genotypes LCT EEN 162/S-1010, LCT EEN 37/I and SIAL93 respectively. Arrows indicate allele gain, slippage and loss. Horizontal axis represents allele size in base pairs. Vertical axis represents peak intensity.

produced by an off-type primary SE (which was not carrying the same mutation), four were from true-to-type primary SEs, and six were from the two missing SEs mentioned above. When screened with the same ten microsatellite markers, the 44 AMAZ 12 secondary SEs exposed to progressive stages of the cryopreservation protocol exhibited no aberrant profiles. 


\section{AGRICULTURAL AND FOOD SCIENCE}

Vol. 18 (2009): 152-159.

\section{SEM analysis}

Although cultured cocoa staminodes (the source of primary SEs) are more extensively callused, embryogenic cotyledonary explants consistently exhibited some callus cells by the stage of embryo initiation. However, all secondary SEs that could be examined in detail appeared to be derived directly from the cotyledon epidermis rather than intermediate callus (Fig. 2).

\section{Discussion}

The passage of cells through in vitro culture may induce perturbation in cellular controls, leading to genomic changes in their regenerants. Processes linked with elevated mutation rates include exposure to auxins, presence of an intermediate callus phase and protacted time in culture: all factors associated with the development of cocoa primary SEs. In the present study primary SEs for a range of cocoa genotypes exhibited pronounced levels of somaclonal variation as evidenced by microsatellite profiles divergent from those of their donor trees. Their descendent secondary SEs showed lower mutation frequencies and an additional population of secondary SEs, produced after a comparable time in culture, then taken through cryopreservation exhibited no mutations when screened with the same microsatellite markers.

The most common polymorphism detected in the present study was microsatellite allele loss. There are several possible causes of such mutations including large deletions causing locus disappearance within the chromosome, total elimination of the chromosome and small indels that prevent primer binding. Changes to the microsatellite sequence can only cause allele loss on rare occasions when slippage gives rise to allele convergence such
Fig. 2. SEM images of cocoa secondary somatic embryos developing on cotyledonary explants from primary somatic embryos (a and c), prior to harvesting for cryopreservation. Solid arrows indicate secondary somatic embryos (shown magnified in $b$ and d) arising from epidermis of cotyledon. $\mathrm{Ca}=$ callus, Ep = epidermis and SSE $=$ secondary somatic embryo. Bars represent $0.5 \mathrm{~mm}$ ( $\mathrm{a}$ and c), $25 \mu \mathrm{m}$ (b) and $150 \mu \mathrm{m}(\mathrm{d})$.
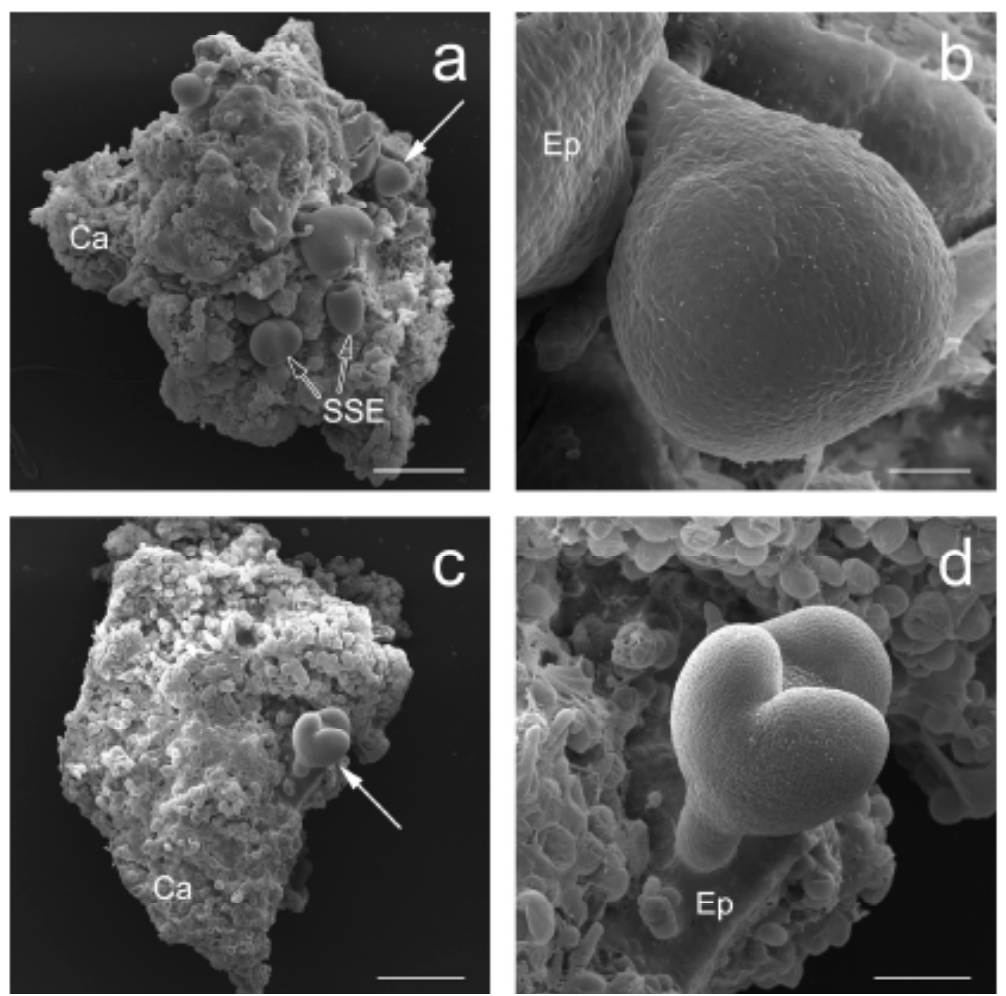
Fang, J-Y. et al. Genetic fidelity of cryopreserved cocoa somatic embryos

that a formerly heterozygous genotype becomes homozygous. This kind of genotype change is, therefore, not related to the microsatellite itself but to the flanking DNA regions. This polymorphic cocoa embling population was further studied to differentiate large scale deletions and chromosome loss from small-scale changes by comparing the behaviour of additional microsatellite markers positioned on the same linkage group (data not shown). These comparisons identified only $7.4 \%$ of the tested samples where two microsatellite markers from the same chromosome were both lost in the same embling (suggesting chromosome loss or large-scale deletions). It follows that most instances of allele loss were probably caused by small-scale events such as indels and point mutations, which accords with studies suggesting that in vitro culture induced chromosomal structural changes are more frequent than numerical changes (Gupta 1998).

Maximova et al. (2002) reported that, while cocoa staminodes gave rise to primary SEs of multicellular origin, embryos from cotyledons developed predominantly from unicellular origins. A similar ontogeny has also been reported for secondary SEs in walnut (Polito et al. 1989) and Korean ginseng (Choi et al. 1997). This distinction may explain the propensity for cocoa primary SEs to form chimeras (Rodriguez-Lopez et al. 2004). It may also be the case that the transition from primary to secondary embryogenesis acts as a screen for mutation transmission: those primary SEs carrying the most debilitating mutational loads may fail to give rise to further SEs and, as the SEM images here illustrate, secondary SE development is not associated with passage through additional callus.

The storage of in vitro plant cultures at low temperatures has been associated with the production of reactive oxygen intermediates (ROI) (Benson and Withers 1987) and subsequent free radical-related damage has been linked to mutagen production (Esterbauer et al. 1988). The treatments used in the cocoa cryopreservation method employed here illicit volatile markers linked to such ROI production (Fang et al. 2008) but, within the limits of the test population used, the present study did not reveal evidence of mutagenesis at any stage of the cryopreservation process. A similar lack of ad- ditional somaclonal variation was noted when the encapsulation-dehydration approach was applied to Digitalis obscura (Sales et al. 2001). The present study did not involve a large cryopreserved population but an additional 39 secondary SEs for the cocoa genotype SPA4 taken through cryopreservation via encapsulation-dehydration and screened with six microsatellite markers covering five linkage groups also failed to exhibit any profiles departing from those of the donor tree.

We propose, therefore, that while cocoa somatic embryogenesis is susceptible to somaclonal variation, the encapsulation-dehydration cryopreservation methodology imposes no significant extra mutational load. Furthermore, there is a pronounced reduction in the off-type frequency of plants derived from secondary rather than primary SEs and we recommend that these are preferentially employed for cryopreservation.

Acknowledgement: This work was supported by a grant from Cocoa Research (UK).

\section{References}

Benson, E.E. \& Withers, L.A. 1987. Gas chromatographic analysis of volatile hydrocarbon production by cryopreserved plant tissue cultures: a non-destructive method for assessing stability. Cryo Letters 8: 35-46.

Choi, Y.E., Yang, D.C., Kim, H.S. \& Choi, K.T. 1997. Distribution and changes of reserve materials in cotyledon cells of Panax ginseng related to direct somatic embryogenesis and germination. Plant Cell Reports 16: 841-846.

Dixit, S., Mandal, B.B., Ahuja, S. \& Srivastava, P.S. 2003. Genetic stability assessment of plants regenerated from cryopreserved embryogenic tissues of Dioscorea bulbifera L. using RAPD, biochemical and morphological analysis. Cryo Letters 24 (2): 77-84.

Esterbauer, H., Zollner, H. \& Schaur, H. 1988. Hydroxyalkenals: cytotoxic products of lipid peroxidation. Biochemistry 1: 311-317.

Fang, J-Y. Wetten, A. \& Hadley, P. 2004. Cryopreservation of cocoa (Theobroma cacao L.) somatic embryos for long-term germplasm storage. Plant Science 166: 669-675.

Fang, J-Y. Wetten, A. \& Johnston, J. 2008. Headspace volatile markers for sensitivity of cocoa ( Theobroma cacao L.) somatic embryos to cryopreservation. Plant Cell Reports 27: 453-461.

Figueira, A. \& Janick, J. 1994. Optimizing carbon dioxide and light levels during in vitro culture of Theobroma ca- 


\section{AGRICULTURAL AND FOOD SCIENCE}

Vol. 18 (2009): 152-159.

cao. Journal of American Society of Horticultural Science 119: 865-871.

Fujimori, S., Washio, T., Higo, K., Ohtomo, Y., Murakami, K., Matsubara, K., Kawai, J., Carninci, P., Hayashizaki, Y. Kikuchi, S. \& Tomita, M. 2003. A novel feature of microsatellites in plants: a distribution gradient along the direction of transcription. Febs Letters 554: 17-22.

Gupta, P.K. 1998. Chromosomal Basis of Somaclonal Variation in Plants. In: Jain SM, Brar DS, Ahloowalia BS, eds. Somaclonal Variation and Induced Mutations in Crop Improvement. Dordrecht, Netherlands: Kluwer p.149-168.

Harding, K. \& Benson, E.E. 2001. The use of microsatellite analysis in Solanum tuberosum L. in vitro plantlets derived from cryopreserved germplasm. Cryo Letters 22: 199-208.

Lanaud, C., Hamon, P. \& Duperray, C. 1992. Estimation of the nuclear DNA content of Theobroma cacao L. by flow cytometry. Café Cacao Thé 36: 3-8.

Lanaud, C., Risterucci, A.M., Pieretti, I., Falque, M., Bouet, A. \& Lagoda, P.J.L. 1999. Isolation and characterisation of microsatellites in Theobroma cacao L. Molecular Ecology 8: 2141-2143.

Liu, Y.G., Liu, L.X., Wang, L. \& Gao, A.Y. 2008. Determination of genetic stability in surviving apple shoots following cryopreservation by vitrification. Cryo Letters 29: 7-14.

Li, Z., Traore, A., Maximova, S. \& Guiltinan, M. 1998. Somatic embryogenesis and plant regeneration from floral explants of cacao (Theobroma cacao L.) using thidiazuron. In Vitro Cell and Developmental Biology-Plant 34: 293-299.

Maximova, S.N., Alemanno, L., Young, A., Ferriere, N., Traore, A. \& Guiltinan, M.J. 2002. Efficiency, genotypic variability, and cellular origin of primary and sec- ondary somatic embryogenesis of Theobroma cacao L. In Vitro Cell and Developmental Biology-Plant 33: 163-172.

Polito, V.S., McGranahan, G., Pinney, C. \& Leslie, C. 1989. Origin of somatic embryos from repetitively embryogenic cultures of walnut (Juglans regia L.): implications for Agrobacterium-mediated transformation. Plant Cell Reports 8: 219-221.

Richards, C.M., Reilley, A., Touchell, D., Antolin, M.F. \& Walters, C. 2004. Microsatellite primers for Texas wild rice (Zizania texana), and a preliminary test of the impact of cryogenic storage on allele frequency at these loci. Conservation Genetics 5: 853-859.

Risterucci, A.M., Grivet, L. N, Goran, J.A.K., Piretti, I., Flament, M.H. \& Lanaud, C. 2000. A high-density linkage map of Theobroma cacao L. Theoretical and Applied Genetics 101: 948-955.

Rodriguez-Lopez, C.M., Wetten, A.C., Wilkinson, M.J. 2004. Detection and quantification of in vitro-culture induced chimerism using simple sequence repeat (SSR) analysis in Theobroma cacao (L.). Theoretical and Applied Genetics 110: 157-166.

Sales, E., Nebauer, S.G., Arrillaga, I. \& Segura, J. 2001. Cryopreservation of Digitalis obscura selected genotypes by encapsulation-dehydration. Planta Medica 67: 833-838.

Turner, S., Krauss, S.L., Bunn, E., Senaratna, T. Dixon, K., Tan, B. \& Touchell, D. 2001. Genetic fidelity and viability of Anigozanthos viridis following tissue culture, cold storage and cryopreservation. Plant Science 161: 1099-1106.

Wilkinson, T., Wetten, A., Prychid, C. and Fay, M.F. 2003. Suitability of cryopreservation for the long term storage of rare and endangered plant species - a case history for Cosmos atrosanguineus. Annals of Botany 91: 65-74. 